\title{
OPEN-LOOP STABILIZATION OF 2D IMPACT JUGGLING
}

\author{
Renaud Ronsse* Philippe Lefèvre ${ }^{* *}$ \\ Rodolphe Sepulchre* \\ * Montefiore Institute B28, Université de Liège \\ Grande Traverse 10, B-4000 Liège, Belgium \\ [R.Ronsse, R.Sepul chre] Qulg.ac.be \\ ** CESAME, Université catholique de Louvain \\ Avenue G. Lemaître 4, B-1348 Louvain-la-Neuve, Belgium \\ lefevre@csam.ucl.ac.be
}

\begin{abstract}
The paper studies the properties of a sinusoidally vibrating wedge billiard as a model for 2D impact juggling. It is shown that some periodic orbits that are unstable in the elastic fixed wedge become exponentially stable in the (non-)elastic vibrating wedge. These orbits are linked with some classical juggling patterns, providing an interesting benchmark for the study of the frequency-locking properties in human rhythmic tasks.
\end{abstract}

Keywords: Open-loop stability, impact control, juggling, discrete-time

\section{INTRODUCTION}

Human juggling is a wide field of investigation for the neuroscientists: it involves eye-hand coordination, inter-limb coordination, learning phases and balancing of several feedback sources (visual, tactile,...) in a complex rhythmic task. These features were mainly studied with the cascade juggling pattern (Beek and Turvey, 1992; Beek and van Santvoord, 1992; van Santvoord and Beek, 1996; Post et al., 2000; Huys and Beek, 2002).

(Sternad, 1999) proposed a comparison between human juggling and a 1D impact dynamical process amenable to mathematical analysis, i.e. the celebrated bouncing ball model, extensively studied in the literature (Holmes, 1982; Guckenheimer and Holmes, 1986; Bapat et al., 1986). Her team explored deeply the human abilities to exploit the open-loop stable solutions inherent to the bouncing ball dynamics with a sinusoidal vibration as control input (Schaal et al., 1996; Sternad et al., 2001a; Sternad et al., 2001b).

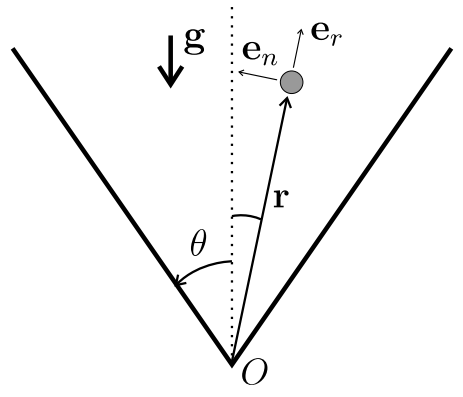

Fig. 1. The wedge billiard.

The 2D wedge billiard (Figure 1) studied in the present paper is viewed as an intermediate between 3D juggling and 1D bouncing ball. It is still amenable to mathematical analysis but presents new features compared to the 1D bouncing ball. The rich dynamical properties of the elastic wedge billiard were first studied in (Lehtihet and Miller, 1986). A model including actuation of the edges has recently been studied by (Sepulchre and Gerard, 2003) for feedback stabilization of (unstable) periodic orbits. (Schaal and Atkeson, 1993) 
mentioned the wedge billiard as an example of juggling device which can stabilize period-1 orbits (see Section 6) using open-loop control.

The present paper uses the model of (Sepulchre and Gerard, 2003) to show the existence of stable period-2 orbits in the (non-)elastic vibrating wedge, i.e. when a sinusoidal motion of the two edges is used to restore the energy dissipated in the impacts. These orbits are unstable in the elastic fixed wedge. In this sense, they are stabilized by the open-loop actuation, even though the stabilizing control requires no feedback.

These orbits are also closely linked to the popular "shower" juggling pattern. For this reason, the wedge billiard provides an interesting benchmark for the study of frequency-locking properties in human rhythmic tasks, close enough to 3D juggling to investigate some critical aspects of human juggling such as spatial configuration of the patterns, inter-limb coordination and synchronization, ...

Section 2 summarizes the actuated wedge billiard model of (Sepulchre and Gerard, 2003). Section 3 shows a qualitative analogy between the square wedge $\left(\theta=45^{\circ}\right)$ and two independent bouncing balls. This analogy is very useful to predict the possible period-2 orbits of the vibrating square wedge and their frequency-locking properties. Section 4 characterizes these orbits and their stability properties in the actuated wedge billiard model. Section 5 links these orbits to popular juggling patterns: 'Site-swap' notation which is used by many jugglers to summarize juggling patterns (Beek and Lewbel, 1995) is proposed to be applicable to the square wedge billiard open-loop patterns. Section 6 characterizes period-1 orbits and their stability in the non-square wedge billiard $\left(\theta \neq 45^{\circ}\right)$.

\section{THE WEDGE BILLIARD MODEL}

The four-dimensional wedge billiard dynamics are studied via the three-dimensional discrete Poincaré map relating the state from one impact to the next one, the ball motion between two impacts being parabolic (a ballistic flight in a constant gravitational field $\mathbf{g})$. Let $\left(\mathbf{e}_{r}, \mathbf{e}_{n}\right)$ an orthonormal frame attached to the fixed point $O$ with $\mathbf{e}_{r}$ aligned with the ball's position vector $\mathbf{r}$ (Figure 1). The ball is assumed to be an unit mass point, let $\mathbf{v}=v_{r} \mathbf{e}_{r}+v_{n} \mathbf{e}_{n}$ denotes its velocity. Following (Lehtihet and Miller, 1986), the state variables are $V_{r}=\frac{v_{r}}{\cos \theta}, V_{n}=\frac{v_{n}}{\sin \theta}$ and $E$, the total energy of the ball, the discrete state vector being:

$$
x[k]=\left(\begin{array}{c}
V_{r}^{+}(t[k]) \\
V_{n}^{+}(t[k]) \\
E^{+}(t[k])
\end{array}\right) \equiv\left(\begin{array}{c}
V_{r}[k] \\
V_{n}[k] \\
E[k]
\end{array}\right)
$$

Where the $\bullet^{+}$notations denote the post-impact values, evaluated at impact time $t[k]$. This is the discrete-time state variables as a convention. The corresponding pre-impact values are denoted $\bullet$.

The uncontrolled billiard map is a composition of a flight map and an impact rule. The flight map is entirely determined by the wedge geometry (that is by the parameter $\alpha=\tan \theta$ ) and does not depend on the gravitational field $g$. It takes the analytical form $\mathcal{F}$ :

$$
\begin{aligned}
& V_{r}(t[k+1])=\left|V_{n}\right|[k] \quad V_{r}[k] \quad f_{1}[k] \\
& V_{n}(t[k+1])=f_{1}[k] \cdot \operatorname{sign}\left(V_{n}[k]\right) \\
& E \quad(t[k+1])=E[k]
\end{aligned}
$$

with

$$
f_{1}[k]=\sqrt{4 E[k]+2 \frac{1}{\left(1+\alpha^{2}\right)^{2}}\left(\left|V_{n}\right|[k] \quad V_{r}[k]\right)^{2} \quad V_{n}^{2}[k]}
$$

when the impacts $k$ and $k+1$ occur on two different edges (Sepulchre and Gerard, 2003), which holds in the steady-state regime of the orbits discussed in this paper.

The impact rule $\mathcal{I}$ simply assumes that the tangential velocity is conserved and that the normal velocity is reversed, proportionally to the coefficient of restitution $e(0<e \leq 1)$, modeling the energy dissipation:

$$
\begin{aligned}
V_{r}[k] & =V_{r}(t[k]) \\
V_{n}[k] & =e V_{n}(t[k]) \\
E[k] & =E \quad(t[k]) \quad\left(1 \quad e^{2}\right) \frac{\alpha^{2}}{2\left(1+\alpha^{2}\right)} V_{n}^{2}(t[k])
\end{aligned}
$$

A simplification that leaves the flight map unchanged is introduced for the controlled wedge billiard, where the discrete control vector consists of the angular deviation $\mu[k]=\mu(t[k])$ of the impacted edge and its angular velocity $\dot{\mu}[k]=\dot{\mu}(t[k])$ (Figure 2, left). The impact rule captures the effect of both the angular velocity control and the angular position control. This simplification rests on the small control assumption: $|\mu| \ll \theta$; and assumes that the impacts still occur on the uncon-
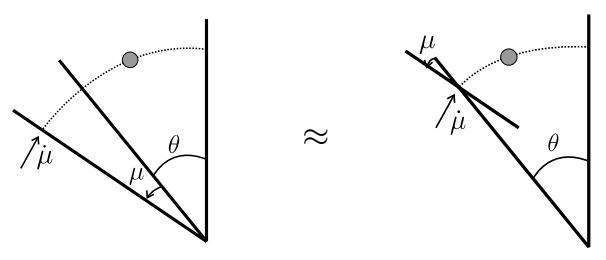

Fig. 2. The controlled wedge billiard (left), and the simplified model when $\mu$ is small (right). 


$$
\begin{aligned}
& \left(\begin{array}{c}
V_{r}[k+1] \\
V_{n}[k+1]
\end{array}\right)=J(\mu[k+1])\left(\begin{array}{cc}
\left|V_{n}\right|[k] & V_{r}[k] \\
f_{1}[k] \cdot \operatorname{sign}\left(V_{n}[k]\right)
\end{array}\right)+\frac{f_{1}[k]}{\alpha}\left(\begin{array}{c}
\alpha \sin \mu[k+1] R[k+1] \\
\cos \mu[k+1] R[k+1]
\end{array}\right) \dot{\mu}[k+1] \\
& R[k+1]=R[k]+\frac{1}{2 g} V_{r}^{2}[k]+\frac{\alpha^{2}}{2 g} V_{n}^{2}[k] \quad \frac{1}{2 g}\left(\left|V_{n}\right|[k] \quad V_{r}[k] \quad f_{1}[k]\right)^{2} \quad \frac{\alpha^{2}}{2 g} f_{1}^{2}[k]
\end{aligned}
$$

trolled wedge but that the angular position control rotates the normal and tangential directions of the impacted edge by an angle $\mu[k]$ (Figure 2, right). The impact rule $\mathcal{I}$ therefore becomes:

$$
\begin{aligned}
M(\mu[k])\left(\begin{array}{c}
V_{r}[k] \\
V_{n}[k]
\end{array}\right)= & \left(\begin{array}{cc}
1 & 0 \\
0 & e
\end{array}\right) M(\mu[k])\left(\begin{array}{l}
V_{r}(t[k]) \\
V_{n}(t[k])
\end{array}\right) \\
& +\left(\begin{array}{c}
0 \\
\frac{1+e}{\alpha} R[k]
\end{array}\right) \dot{\mu}[k]
\end{aligned}
$$

with $M(\mu)$ denoting the rotation matrix:

$$
M(\mu)=\left(\begin{array}{cc}
\cos \mu & \alpha \sin \mu \\
\frac{\sin \mu}{\alpha} & \cos \mu
\end{array}\right)
$$

and $R[k]=\frac{r(t[k])}{\cos \theta}$ being the ball position at impact $k$. It is obtained from the energy equation:

$$
E[k]=\frac{1}{1+\alpha^{2}}\left(\frac{1}{2} V_{r}^{2}[k]+\frac{\alpha^{2}}{2} V_{n}^{2}[k]+g R[k]\right)
$$

The wedge billiard map $\mathcal{B}$ is therefore given by the equation (5) with

$$
\begin{aligned}
J(\mu) & =M(\quad \mu)\left(\begin{array}{cc}
1 & 0 \\
0 & e
\end{array}\right) M(\mu) \\
& =\left(\begin{array}{ccc}
\cos ^{2} \mu & e \sin ^{2} \mu & \frac{\alpha(1+e)}{2} \sin 2 \mu \\
\frac{1+e}{2 \alpha} \sin 2 \mu & \sin ^{2} \mu & e \cos ^{2} \mu
\end{array}\right)
\end{aligned}
$$

Instead of the energy, the impact position $R[k]$ is a more relevant third state variable for the control applications discussed in this paper. Its update is directly derived from the energy equation (9) and is given by the equation (6).

The flight time (time elapsed between two successive impacts) is given by the following equation:

$$
\begin{array}{rlr}
\Delta t[k+1] & =t[k+1] & t[k] \\
& =\frac{1}{g}\left(\begin{array}{lll}
f_{1}[k] & \left.\frac{(1}{1} \alpha^{2}\right)\left|V_{n}\right|[k] & 2 V_{r}[k] \\
1+\alpha^{2}
\end{array}\right)
\end{array}
$$

\section{A BOUNCING BALL APPROXIMATION OF THE VIBRATING SQUARE WEDGE BILLIARD}

The input considered in this paper is a sinusoidal vibration on both edges, directly inspired by the extensively studied bouncing ball system (Holmes, 1982; Guckenheimer and Holmes, 1986; Bapat et al., 1986). A ball bouncing on a sinusoidally vibrating table sustains the simple period-1 motion (constant rebounds) as an exponentially stable motion in a range of the vibration parameters (amplitude and frequency). This period-1 motion is characterized by a frequency-locking relation with the table: the ball period is a multiple of the table period.

The square wedge billiard ( $\alpha=1)$ is a very special case of the general wedge billiard. In the absence of control, the 2 DOF motion decouples into two 1 DOF independent motions. The normal velocity at impact can be expressed as $V_{n}[k+2]=e V_{n}[k]$, exhibiting the decoupling between the dynamics along each edge. In a first (crude) approximation of the model, it is convenient to disregard the coupling introduced by the input and to view the two edges as two anti-phase vibrating tables (one is up when the other is down). The open-loop stability properties of the bouncing ball are then transposed to the two decoupled motions of the square wedge billiard. In the steady-state regime, two frequency-locking relations will exist between the ball an the wedge:

$$
\begin{aligned}
& (t[k+2] \quad t[k])^{\star}=n \frac{2 \pi}{\omega} \\
& (t[k+1] \quad t[k])^{\star}=\left(\begin{array}{ll}
2 m & 1
\end{array}\right) \frac{\pi}{\omega}
\end{aligned}
$$

where $\omega$ denotes the vibration frequency and • denotes the steady-state solutions. These relations rest on the trivial assumption $m \leq n, m$ and $n$ being positive integers. Equation (12) transposes the frequency-locking relation of the bouncing ball on each dynamics. Equation (13) means that the time between two successive impacts must be equal to an odd multiple of the vibration half-frequency, the edges vibrating at the same

Table 1. Periodic orbits for the square wedge billiard. $(x: y)$ denotes the ratio between the low toss and the high toss flight time.

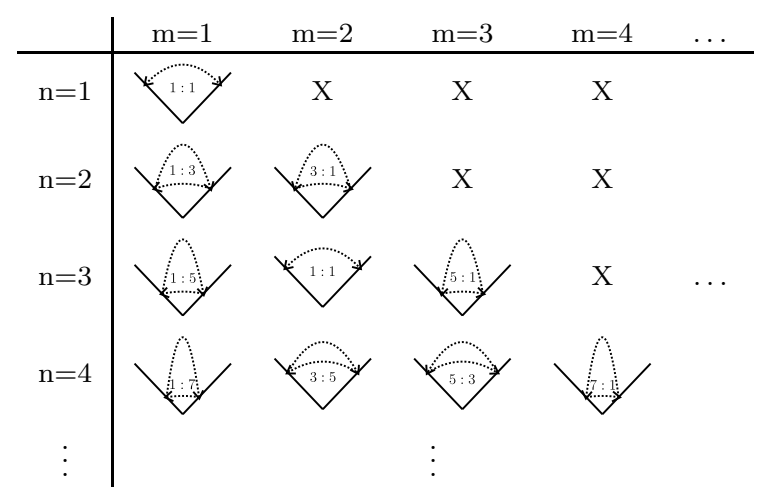




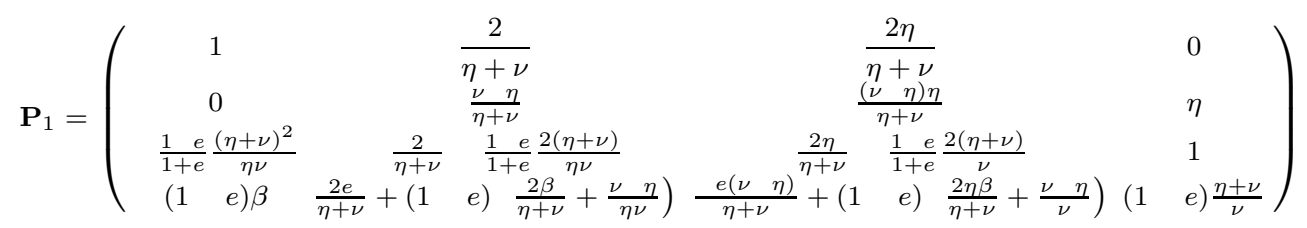

frequency. As a convention, $m$ will be associated with the flight time between the right edge and the left edge (the "low toss"), then the flight time between the left edge and the right edge (the "high toss") will be $(2 n \quad 2 m+1) \frac{\pi}{\omega}$ in the steady-state regime.

Table 1 illustrates the first periodic orbits for the vibrating square wedge billiard and the ratio between the low toss and the high toss flight time for each of these orbits. The stability properties of these orbits are immediately inherited from the decoupled dynamics. A period-1 motion is exponentially stable for the bouncing ball if:

$$
n \pi \frac{1 \quad e}{1+e}<\frac{A \omega^{2}}{g}<\sqrt{n^{2} \pi^{2}\left(\frac{1}{1+e}\right)^{2}+1}
$$

where $A$ and $\omega$ are the vibration amplitude and frequency (Holmes, 1982). This inequality holds if a "small amplitude" assumption is made. (Bapat et al., 1986) studied and quantified this assumption. Significant differences between the exact and the approximated model are more likely if $e$ is below about 0.8 , but the approximated model provides a good description of the qualitative dynamical behavior.

\section{STABLE PERIODIC ORBITS IN THE VIBRATING SQUARE WEDGE MODEL}

The presence of a sinusoidal input in the square wedge billiard model of Section 2 couples the bouncing ball dynamics of Section 3 for two reasons:

- The energy restored to the ball at each impact depends on the impact position. This position is influenced by the other edge.

- The post-impact velocities depend on the control angle $\mu$ which itself depends on the flight time between two successive impacts.

However this coupling does not destroy the periodic orbits studied in Section 3. Injecting the steady-state time relations (12) and (13) into the dynamical equation (11) (with $\alpha=1$ ), the steadystate solutions are:

$$
\begin{aligned}
\left|V_{n}\right|^{\star} & =\frac{n \pi g}{\omega} \\
\left(V_{r}[2 k]\right)^{\star} & =\frac{(n 2 m+1) \pi g}{\omega} \\
& =\left(V_{r}[2 k+1]\right)^{\star}
\end{aligned}
$$

with the even impacts occurring on the right edge as a convention. The steady-state value of $R$ is derived from (3) and (9):

$$
R^{\star}=\frac{g}{2}\left(\frac{\pi}{\omega}\right)^{2}\left(\begin{array}{lll}
2 m & 1
\end{array}\right)(2 n \quad 2 m+1)
$$

Conservation of the normal velocity at impact times gives the steady-state edges velocity:

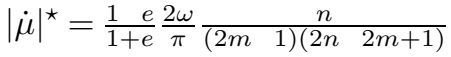

$$
\begin{aligned}
& \Rightarrow \cos (\omega t[2 k])^{\star}=\cos \varphi^{\star} \\
& =\frac{1}{1+e} \frac{2}{A \pi} \frac{n}{(2 m \quad 1)(2 n \quad 2 m+1)} \\
& =\cos (\omega t[2 k+1])^{\star}
\end{aligned}
$$

whereas the steady-state edge position is given by the fixed square wedge configuration. The small control approximation is done around the steadystate solution. The input is actually an offset sinusoid:

$$
\begin{gathered}
\mu[k]=A\left(\sin (\omega t[k]) \quad \sin \varphi^{\star} \operatorname{sign}\left(V_{n}[k]\right)\right) \\
\dot{\mu}[k]=A \omega \cos (\omega t[k])
\end{gathered}
$$

Therefore, the linearized control inputs are:

$$
\begin{aligned}
\delta \mu[k] & =A \omega \cos \varphi^{\star} \operatorname{sign}\left(V_{n}[k]\right) \delta t[k] \\
\delta \dot{\mu}[k] & =A \omega^{2} \sin \varphi^{\star} \operatorname{sign}\left(V_{n}[k]\right) \delta t[k] \\
& =\frac{1}{1+e} \frac{\omega^{2}}{\pi^{2}} \operatorname{sign}\left(V_{n}[k]\right) \delta t[k]
\end{aligned}
$$

with

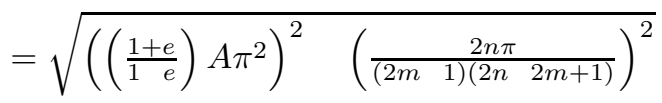

Stability of the linearized system is now studied with an dimensionless state vector:

$$
\mathbf{x}^{\prime}[k]=\left(\begin{array}{c}
\tau[k] \\
\rho[k] \\
W_{r}[k] \\
\left|W_{n}\right|[k]
\end{array}\right)=\left(\begin{array}{c}
\omega t[k] \\
\frac{\omega^{2}}{\pi g} R[k] \\
\frac{\omega}{g} V_{r}[k] \\
\frac{\omega}{g}\left|V_{n}\right|[k]
\end{array}\right)
$$

The Jacobian matrices are obtained by a linearization of the equations (11), (6) and (5):

$$
\delta \mathbf{x}^{\prime}[k+2]=\mathbf{P}_{2} \delta \mathbf{x}^{\prime}[k+1]=\mathbf{P}_{2} \mathbf{P}_{1} \delta \mathbf{x}^{\prime}[k]
$$

$\mathbf{P}_{1}$ is given by (14) with

$$
\beta=\frac{(\nu+\eta)(\eta \quad \nu)}{\nu \eta} \quad \frac{\nu \eta}{2}
$$

where $\eta=2 m \quad 1$ and $\nu=2 n \quad 2 m+1$ are linked with the steady-state flight times. $\mathbf{P}_{2}$ has 


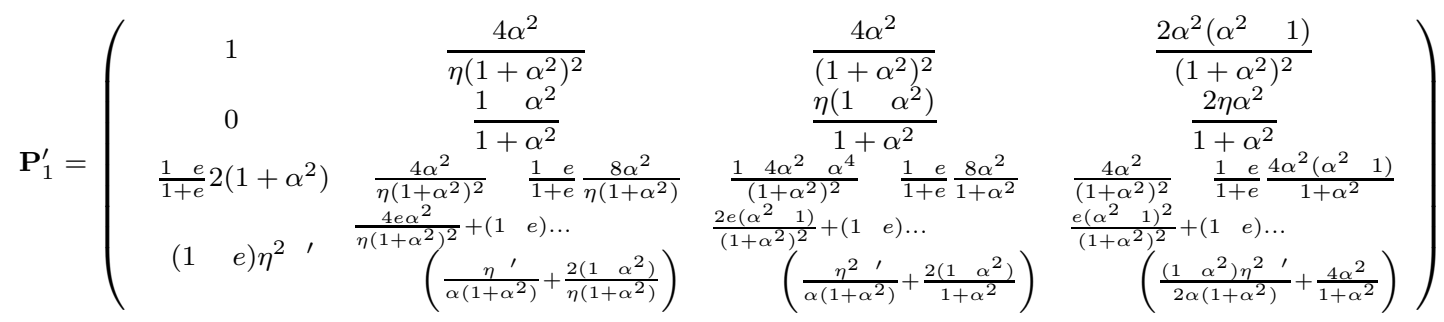

exactly the same structure as $\mathbf{P}_{1}$ with an exchange between $\nu$ and $\eta$.

The eigenvalues of $\mathbf{P}_{2} \mathbf{P}_{1}$ were numerically computed. Figure 3 represents the stability region for the simple $(1: 1)$ orbit $(n=m=1)$ and the $(1: 3)$ orbit $(n=2, m=1)$. This is the region where the absolute values of the four eigenvalues of $\mathbf{P}_{2} \mathbf{P}_{1}$ are less than 1 . The stability area is dramatically reduced for the (1:3) orbit. Actually, the larger $n$, the smaller the stability area. Nevertheless, each periodic orbit is exponentially stable, as in the "double bouncing ball" model. In contrast, all these orbits are unstable for the uncontrolled elastic square wedge billiard (Sepulchre and Gerard, 2003).

\section{A LINK TO IMPACT JUGGLING}

According to the stability conditions discussed in Section 3, $n$ balls can be "juggled" in the square wedge billiard configuration, with a temporal delay of $\frac{2 \pi}{\omega}$ between each ball. In this case, the impacts will happen on equally spaced beats. The topology of the characterized periodic orbits with $n$ balls is the popular $n$ balls "juggling shower": one hand throws the balls in a high arc over to the other hand and the second hand passes the balls very quickly back to the first hand. Temporal ratio between the throws in the shower are the same as in the periodic orbits for the square wedge billiard: (1:3) with 2 balls, $(1: 5)$ with 3 balls,..

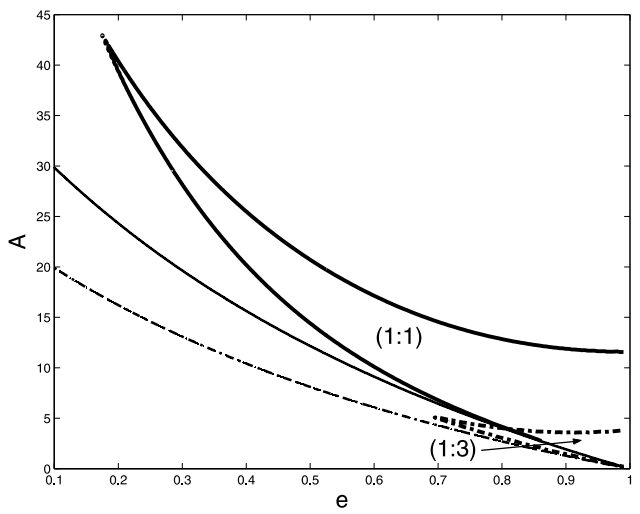

Fig. 3. Stability region of two periodic orbits: $(1: 1): n=1, m=1$ (solid line); $(1: 3)$ : $n=2, m=1$ (dashdotted line). The thinner lines represent the physical minimum limit for the amplitude A (equation (22)).
Site-swaps notation (Beek and Lewbel, 1995) is a compact notation representing the order in which props are thrown and caught in each cycle of the juggle, assuming throws happen on beats that are equally spaced in time. The site-swap notation of the 3 balls shower is simply ' 51 ', where the 5 refers to the duration of the high toss and the 1 to the time needed to pass the ball from one hand to the other on the lower part of the arc. Likewise with each $(x: y)$ orbit presented in Table 1: its site-swap notation will be ' $x y$ '.

The edges could therefore be viewed as an idealization of the juggler's two arms, assuming that each catch and its subsequent throw are instantaneous (impact juggling).

\section{AROUND THE SQUARE WEDGE BILLIARD: $\alpha \neq 1$}

For any $\alpha \neq 1$, the uncontrolled elastic wedge billiard has a $(1: 1)$ solution, characterized by $\left|V_{n}\right|^{\star}=f_{1}^{\star}$ and $V_{r}^{\star}=0$. In contrast, no period2 orbit exists in the elastic nonsquare wedge. Period-2 orbits could exist in the (non-)elastic vibrating wedge when $\alpha \neq 1$ but require braking the symmetry of the impacts. This will be the subject of a future publication: some preliminary results exhibit such a solution in a thin domain around the square configuration.

The (1: 1$)$ solution $(n=2 m \quad 1)$ is easy to calculate for the (non-)elastic vibrating wedge billiard:

$$
\begin{aligned}
\left|V_{n}\right|^{\star} & =\frac{1+\alpha^{2}}{\alpha^{2}} \frac{(2 m \quad 1) \pi g}{2 \omega} \\
\left|\cos \varphi^{\star}\right| & =\alpha \frac{1}{1+e} \frac{2}{A \pi} \frac{1}{(2 m \quad 1)}
\end{aligned}
$$

The stability of this solution is analyzed by a linearization method: $\delta \mathbf{x}^{\prime}[k+1]=\mathbf{P}_{1}^{\prime} \delta \mathbf{x}^{\prime}[k]$ with $\mathbf{P}_{1}^{\prime}$ given by the equation (32) where

$$
'=\sqrt{\left(\left(\frac{1+e}{1}\right) A \pi^{2}\right)^{2}\left(\frac{2 \pi \alpha}{2 m i}\right)^{2}}
$$

Figure 4 represents the stability region when $e=$ 0.9 and $e=0.5$. The larger the dissipation, the thinner the stability region. This analysis agrees with the previous analysis of (Schaal and Atkeson, 1993). 


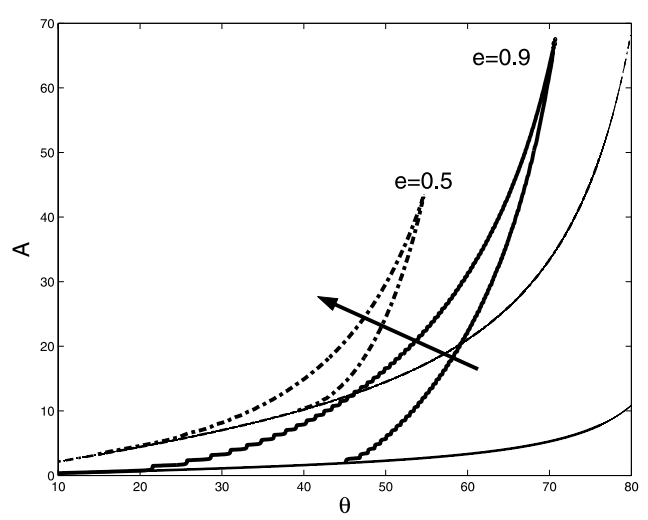

Fig. 4. Stability region the $(1: 1)$ orbit: $e=0.9$ (solid line); $e=0.5$ (dashdotted line). The thinner lines represent the physical minimum limit for the amplitude A (equation (34)). The arrow denotes the effect of a decreasing coefficient of restitution $e$.

\section{CONCLUSION}

This paper presents the sinusoidally vibrating wedge billiard as an interesting benchmark for the study of human rhythmic skills. Open-loop stable orbits were characterized for the particular case of the square wedge billiard $\left(\theta=45^{\circ}\right)$. These orbits are governed by a temporal-locking relation similar with a popular juggling pattern (the shower). $(1: 1)$ orbits exist and are stable over a wide range of wedge angle, while the other orbits exist only around the square wedge configuration. Further research will explore the human abilities to exploit these stability properties in juggling skill.

\section{ACKNOWLEDGEMENTS}

This paper presents research partially supported by the Belgian Programme on Interuniversity Attraction Poles (IAP v/22), initiated by the Belgian State, Prime Minister's Office for Science, Technology and Culture.

\section{REFERENCES}

Bapat, C.N., S. Sankar and N. Popplewell (1986). Repeated impacts on a sinusoidally vibrating table. J. Sound Vibration 108(1), 99-115.

Beek, P. J. and A. A. M. van Santvoord (1992). Learning the cascade juggle - a dynamicsystems analysis. J Mot Behav 24(1), 85-94.

Beek, P. J. and A. Lewbel (1995). The science of juggling. Scientific American 273(5), 92-97.

Beek, P. J. and M. T. Turvey (1992). Temporal patterning in cascade juggling. J Exp Psychol Hum Percept Perform 18(4), 934-47.

Guckenheimer, J. and P. J. Holmes (1986). Nonlinear oscillations, dynamical systems and bifurcations of vector fields. Springer-Verlag. New York.
Holmes, P. J. (1982). The dynamics of repeated impacts with a sinusoidally vibrating table. J. Sound Vibration 84(2), 173-189.

Huys, R. and P. J. Beek (2002). The coupling between point-of-gaze and ball movements in three-ball cascade juggling: the effects of expertise, pattern and tempo. J Sports Sci $\mathbf{2 0}(3), 171-86$.

Lehtihet, H. E. and B. N. Miller (1986). Numerical study of a billiard in a gravitational field. Phys. D 21(1), 93-104.

Post, A. A., A. Daffertshofer and P. J. Beek (2000). Principal components in three-ball cascade juggling. Biol Cybern 82(2), 143-52.

Schaal, S. and C. G. Atkeson (1993). Open loop stable control strategies for robot juggling. In: IEEE International Conference on Robotics and Automation. Vol. 3. pp. 913-918.

Schaal, S., C. G. Atkeson and D. Sternad (1996). One-handed juggling: A dynamical approach to a rhythmic movement task. J Mot Behav $\mathbf{2 8}(2), 165-183$.

Sepulchre, R. and M. Gerard (2003). Stabilization of periodic orbits in a wedge billiard. In: $42 n d$ IEEE Conference on Decision and Control. Hawaii.

Sternad, D. (1999). Juggling and bouncing balls: Parallels and differences in dynamic concepts and tools. International Journal of Sport Psychology 30(4), 462-489.

Sternad, D., M. Duarte, H. Katsumata and S. Schaal $(2001 a)$. Bouncing a ball: tuning into dynamic stability. J Exp Psychol Hum Percept Perform 27(5), 1163-84.

Sternad, D., M. Duarte, H. Katsumata and S. Schaal (2001b). Dynamics of a bouncing ball in human performance. Physical Review E $6301(1)$, art. no.-011902.

van Santvoord, A. A. M. and P. J. Beek (1996). Spatiotemporal variability in cascade juggling. Acta Psychologica 91(2), 131-151. 